\title{
Modified thoracolumbar interfascial plane block versus epidural analgesia at closure for lumbar discectomy: a randomized prospective study
}

\author{
Erkan Cem Çelik, MD ${ }^{1}$, Mursel Ekinci, MD2, Ahmet Murat Yayik, MD ${ }^{1}$ \\ Ali Ahiskalioglu, MD³, Muhammed Enes Aydi, MD, Nuh Cagri Karaavci, MD ${ }^{4}$ \\ 1. Assistant Professor, Atatürk University, Medical Faculty, Department of Anesthesiology and Reanimation, Erzurum,TURKEY. \\ 2. Assistant Professor, Bursa City Hospital, Department of Anesthesiology and Reanimation, Bursa, TURKEY \\ 3. Associate Professor, Atatürk University, Medical Faculty, Department of Anesthesiology and Reanimation, Erzurum, TURKEY. \\ 4. Assistant Professor, Atatürk University, Medical Faculty, Department of Neurosurgery, Erzurum, TURKEY.
}

Correspondence: Erkan Cem ÇELIK, Assistant Professor, Department of Anesthesiology and Reanimation, Medical Faculty, Atatürk University, Erzurum, Turkey; E-mail: drerkancem@yahoo.com; Phone: +90 5053442181

\section{Abstract}

Background \& objective: The postoperative period of lumbar discectomy surgery usually involves a period of moderate to severe pain if adequate pain management is not practiced. Various pain controlling methods have been used other than oral and/or parenteral analgesic administration. We aimed to examine the effect of epidural analgesia at closure (EAC) versus modified thoracolumbar interfascial plane (mTLIP) block on postoperative opioid consumption in patients undergoing lumbar discectomy.

Methodology: It was a randomized, prospective study involving sixty adult patients undergoing single-level lumbar discectomy. Patients were randomly assigned to two groups. mTLIP group $(n=30)$ received ultrasound-guided bilateral mTLIP block with $20 \mathrm{ml}$ of $0.25 \%$ bupivacaine. EAC group $(n=30)$ received $20 \mathrm{ml}$ of $0.25 \%$ bupivacaine to the epidural space by the surgical team at the closure stage of surgery. Postoperatively, analgesia was performed with intravenous tramadol with a patient-controlled analgesia (PCA) pump. Visual analog scale (VAS) scores, opioid consumption, rescue analgesia and side effects were recorded.

Results: Groups had similar demographic measures. There was statistically no difference in terms of opioid consumption from zero to 4 th $\mathrm{hr}$ and VAS scores in the 1-2 hrs postoperatively $(p>0.05)$ between groups. At 4-12 hrs and 12-24 postoperatively hrs intervals, total opioid consumption was significantly lower in Group mTLIP compared to Group EAC $(p<0.05)$. At the 4th, 8th, 12th, and 24th hrs VAS scores were lower in Group mTLIP compared to Group EAC $(p<0.05)$. Rescue analgesia usage was significantly higher in the Group EAC than in the Group mTLIP, e.g. $11 / 30$ vs. $3 / 30$ respectively $(p=0.015)$.

Conclusion: Preoperative bilateral, ultrasound-guided modified thoracolumbar interfascial plane block offers more effective postoperative analgesia, thus reducing tramadol consumption as compared to epidural analgesia at closure after lumbar discectomy surgery.

Key words: Thoracolumbar interfascial plane block; Epidural analgesia'; Lumbar discectomy; Postoperative pain; Ultrasound guidance

Citation: Çelik EC, Ekinci M, Yayik AM, Ahiskalioglu A, Aydi ME, Karaavci NC. Modified thoracolumbar interfascial plane block versus epidural analgesia at closure for lumbar discectomy: a randomized prospective study. Anaesth. pain intensive care 2020;24(6):588-595; DOI: 10.35975/apic.v24i5.1354

Abbreviations: EAC - Epidural analgesia at closure; TLIP - thoracolumbar interfascial plane; mTLIP - modified thoracolumbar interfascial plane 


\section{Introduction}

Disc herniation is described as the displacement of the disc material from the intervertebral disc space. ${ }^{1}$ It is more common in the cervical and lumbar regions. ${ }^{2}$ The incidence of lumbar disc herniation in different societies varies between 1-3\%.-5 Patients operated upon for the pain caused by lumbar disk herniation may suffer from insufficient postoperative analgesia leading to delayed mobilization, increased incidence of thromboembolism, and prolonged duration of hospital stay in the postoperative period. ${ }^{6}$. Moreover, if the pain is not reduced or alleviated to tolerable levels in the early period, it can lead to the development of chronic pain. ${ }^{7}$

Owing to surgical trauma, spinal surgery can be followed by pain originating in the vertebra, intervertebral disks, facet joints, muscles, skin and subcutaneous tissues. ${ }^{8}$ Although pain is of nociceptive origin, it can also follow a pattern of neuropathic pain. ${ }^{9}$ Therefore, adequate pain relief following spinal surgery is utmost essential. It can include the use of non-steroidal anti-inflammatory drugs, gabapentin, pregabalin, systemic opioids, lidocaine infusion, neuraxial analgesia and regional blocks that have become popular in the recent years. ${ }^{9-11}$

The thoracolumbar interfascial plane (TLIP) block was defined by Hand et al. for reducing pain following spinal surgery. In this block, the anesthetic drug is administered between the multifidus and longissimus muscles to target the dorsal ramus of the thoracolumbar nerves. ${ }^{12}$ The TLIP block was later modified by Ahiskalioglu et al., with the involvement of the administration of a local anesthetic between the longissimus and iliocostalis, which was observed to demonstrate a dermatomal dispersion similar to that by the TLIP block. ${ }^{13}$

Our hypothesis was that the patients who received a pre-operative bilateral modified TLIP block (mTLIP) would require less tramadol and have lower pain scores than those who received an epidural analgesia at closure (EAC) for lumbar discectomy surgery in the setting of multimodal analgesia. Our primary outcome was total tramadol consumption during the $24 \mathrm{hrs}$ after surgery. Secondary outcomes included postoperative pain severity, side effects of the blocks and the opioids.

\section{Methodology}

The study design was approved by the institutional ethics committee. This randomized clinical trial recruited 60 patients aged between 18 and 70 yrs with the American Society of Anesthesiologists (ASA) physical status I/II who were candidates for surgical operations on single level lumbar discectomy. All the participants were asked to sign an informed consent form after having been provided with details of the aim and proceedings of the study. Patients were excluded from the study if they had a neurological and neuromuscular disorders, psychiatric problems, cardiopulmonary diseases, coagulopathy, infections or allergy to local anesthetic agents, a previous history of opioid use or opioid abuse, chronic nonsteroidal antiinflammatory drugs usage. Patients were divided into two groups as Group mTLIP and Group EAC by computer program for randomization.

20 gauge cannulas were placed over the left hands of the patients in the regional anesthesia room and 4 $\mathrm{ml} / \mathrm{kg} 0.9 \%$ saline infusion was started. The age, weight and sex of the patients were recorded and the ECG, $\mathrm{SpO}_{2}$, non-invasive blood pressure were monitored. In Group mTLIP, modified TLIP block was applied before surgery in the regional anesthesia room. In Group EAC, epidural anesthesia was applied by the operating surgeon at closure.

Patients in the Group mTLIP were taken to the local anesthesia room for administering the modified TLIP block. Once in the anesthesia room, they were placed in the prone position. The area identified at L4 and its surroundings were cleaned with povidone-iodine and covered with sterile drapes. The ultrasound probe prepared in a sterile manner, was placed on the fourth vertebra in the midline position on the transverse line. (Figure 1-A) The locations of the spinous processes and interspinal muscles were identified. The transverse process was visualized by moving/sliding the probe slightly to the lateral side. The multifidus, longissimus and iliocostalis muscles on the transverse process were identified (Figure 1-B). After the skin was anesthetized with $1 \%$ lidocaine, intervention with an 80-mm 21G SonoPlex ${ }^{\mathrm{TM}}$ block needle (Pajunk ${ }^{\circledR}$ Medical Inc.) was initiated in the medial-lateral position with the assistance of ultrasonography (USG). The area between the longissimus and 


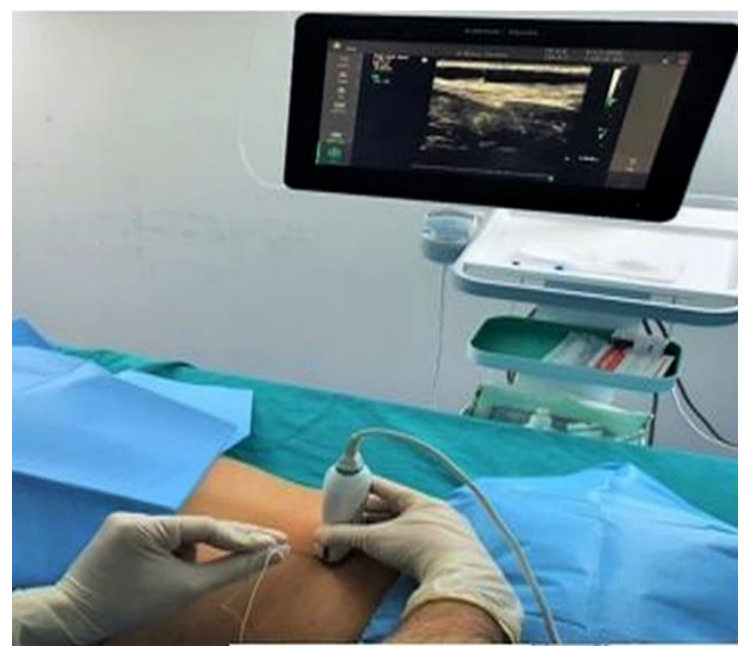

Figure 1-A: USG probe at midline position on the transverse line

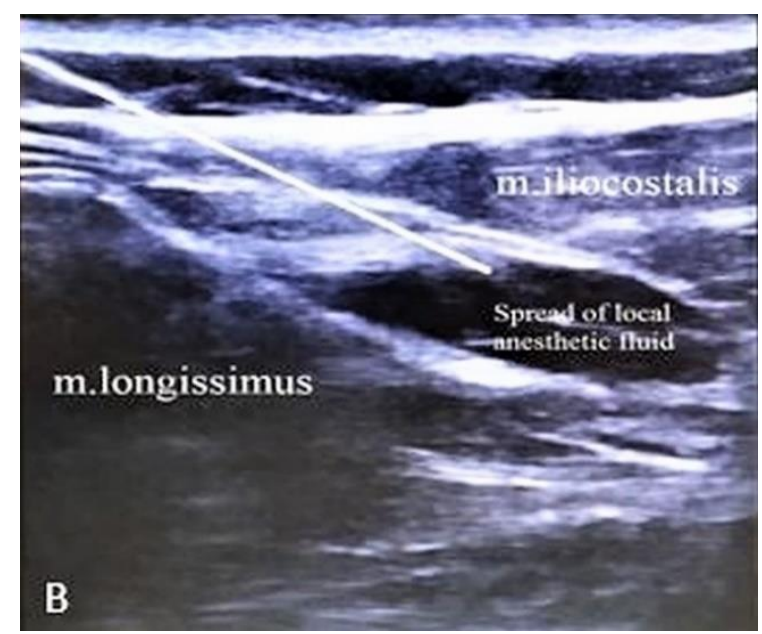

Figure 1-B: Identification of the three muscles

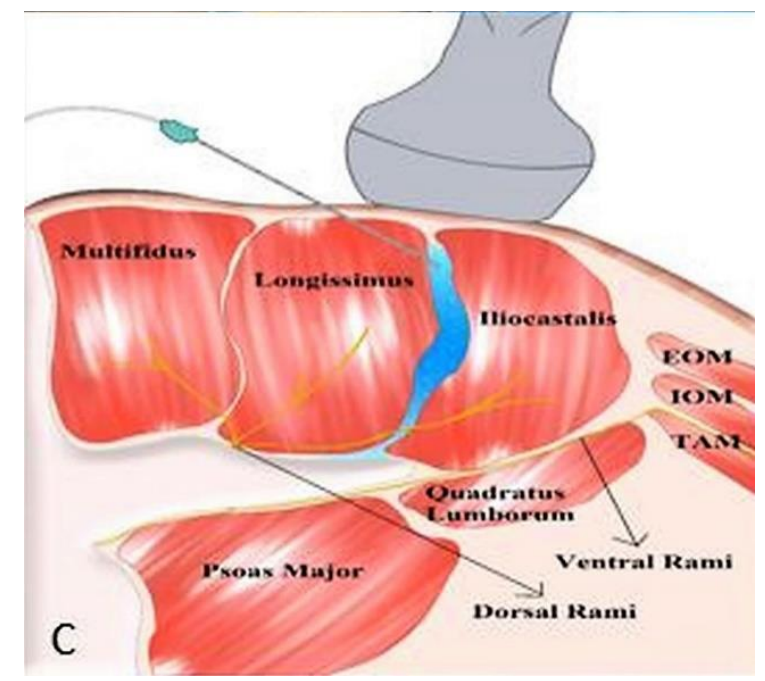

Figure 1-C Block needle between the longissimus and iliocostalis muscles iliocostalis muscles was visualized, and the block needle was inserted between them. (Figure 1-C) No blood was observed after negative aspiration, and the location was confirmed by applying hydrodissection with $2 \mathrm{ml}$ saline. Following this, $20 \mathrm{ml}$ of $0.25 \%$ bupivacaine (without epinephrine) was applied between the fasciae of two muscles. The same procedure was performed on the other side. Block effectiveness was assessed at 20th min by hot-cold discrimination and pin-prick tests. Routine general anesthesia was induced in both groups with $2 \mathrm{mg} / \mathrm{kg}$ propofol, $50 \mathrm{mcg}$ fentanyl and $0.6 \mathrm{mg} / \mathrm{kg}$ rocuronium. Anesthesia was maintained with $2 \%$ sevoflurane in $40 \% \mathrm{O}_{2}$ and $60 \%$ air. Remifentanil infusion was started at $0.05-0.1 \mu \mathrm{g} / \mathrm{kg} / \mathrm{min}$. All patients underwent open discectomy, performed by the same surgeon using the same surgical technique. In the Group EAC patients, the epidural block was administered by the same surgical team at the end of the surgical procedure through the application of $20 \mathrm{ml}$ of $0.25 \%$ bupivacaine to the epidural space after hemostasis was achieved and before the fascia and subcutaneous tissue closure was started. The closure procedure was applied immediately following administration of the epidural anesthesia to reduce the leakage/escape of the local anesthetic solution outside the epidural area. Patients was administered $0.01 \mathrm{mg} / \mathrm{kg}$ atropine iv and 0.02 $\mathrm{mg} / \mathrm{kg}$ neostigmine and extubated at the end of the surgery and were taken to post anesthesia care unit (PACU).

For postoperative analgesia, PCA was used to administer a loading dose of $50 \mathrm{mcg}$ tramadol and 10 $\mathrm{mg}$ on demand with 20 min lock-out interval. Inj. meperidine $25 \mathrm{mg}$ was used as rescue analgesia in patient with visual analog scale (VAS) score above 4. Opioid consumption in the postoperative periods of 0 4, 4-12, and 12-24 hrs, side effects and VAS scores in the postoperative 1 st 2 nd, 4 th, 8 th, 12th and 24 th hrs were recorded.

\section{Sample Size}

The post-hoc sample size calculation was based on the primary outcome variable; total tramadol consumption in the first $24 \mathrm{hrs}$ after surgery. Calculations were done with the G*Power program (Heinrich-HeineUniversity, Düsseldorf, Germany). The effect size was calculated from our findings. It was determined that the effect size was 1.21 in the $95 \%$ confidence interval, alpha error of 0.05 , and the power was 0.99 in the 
significance level. This result indicates that the study sample size was sufficient.

\section{Statistical Analysis}

IBM SPSS 20.0 software program was used to perform the statistical analysis. Data were analyzed by using the Kolmogorov-Smirnov test for distribution of valuable. Pearson $\chi 2$ test and fisher's exact test were used to compare categorical data between groups. Differences among groups were controlled by using the Student's t-test at a significance level of 5\%. Descriptive statistics was explained as mean \pm standard deviation (SD).

\section{Results}

In this study, 30 patients were included in mTLIP group, and 31 patients in EAC group. One patient in EAC group was excluded from study owing to changing surgical technique. Data for mTLIP and EAC groups, each consisting of 30 patients, were subjected for statistical analysis (Figure 2). No statistical difference was observed between the groups with respect to age, gender, weight and height, level of surgery and duration of surgery $(p>0.05)$ (Table 1).

A comparison of opioid consumption between the two groups revealed that although there was no statistically significant difference in terms of opioid consumption between 0-4 hrs postoperatively ( $p>0.05$ ), the total opioid consumption was significantly lower in Group mTLIP between 4 th and 12th hrs and hrs 12th and 24th $(\mathrm{p}<0.05)$ (Table 2).

In the postoperative recovery room following the surgery, no statistically significant difference was observed between the mTLIP and EAC Groups in terms of the VAS scores in the postoperative hrs $1^{\text {st }}$ and $2^{\text {nd }}(\mathrm{p}>0.05)$, whereas the VAS scores at hrs $4^{\text {th }}$, $8^{\text {th }}, 12^{\text {th }}$ and $24^{\text {th }}$ were statistically significantly lower in the mTLIP Group ( $\mathrm{p}<0.05)$ (Table 3).

Additional need for rescue analgesics was observed in 11 patients in the EAC Group and in 3 patients in the mTLIP Group, and it was found that the difference between the groups was statistically significant $(\mathrm{p}<$ 0.05) (Table 2). An evaluation of the postoperative complications showed that there were no breathing depression, sedation/confusion and somnolence in either groups.
There was no statistical significant difference between the two groups with respect to nausea/vomiting, pruritus, urinary retention and constipation $(\mathrm{p}>0.05)$ (Table 4).

\section{Discussion}

In this study our hypothesis was that patients who received a pre-operative bilateral modified TLIP block would require less tramadol and have lower pain scores than those who received epidural analgesia at closure for lumbar discectomy surgery in the setting of multimodal analgesia. Based on the study results, it was found that patients who were administered the modified TLIP block had lower total opioid consumption and pain scores for $24 \mathrm{hrs}$ than patients who were administered epidural analgesia at closure.

There are numerous studies reporting on the effectiveness of local anesthetic agents or steroids administered at the end of lumbar decompression surgery to the epidural space without leaving a catheter. ${ }^{6,14}$ Although continuous techniques are used in cases of back surgery through catheter management, we preferred to administer a single-dose injection in this study to avoid risks such as dural puncture, paresthesia, neurological damage and paraplegia that might be caused by catheters and based on a suspicion that neurological deficits secondary to the surgery might be overlooked. ${ }^{15}$

It is believed that the primary area of effect of local anesthetics that are administered in the epidural space, is the dorsal and ventral nerve roots exiting the spinal column. Although epidural administered local anesthetics can lead to sensorial and motor block depending on the dose, we aimed to induce the sensorial block without causing motor block by applying a single dose of epidural analgesia in this study. ${ }^{14}$ In recent years, ultrasound-guided blocks have become one of the preferred methods for pain control undergoing spinal surgery, and numerous cases of this have been reported. ${ }^{16-18}$

The effectiveness of the thoracolumbar interfacial plane block and erector spinae plane block has been demonstrated both by our study and randomized controlled studies. These blocks have been reported to significantly decrease opioid consumption, use of rescue analgesia and incidence of side effects. ${ }^{11,19,20}$ 
Table 1: Comparison of patients' characteristics, surgical duration and level of surgery

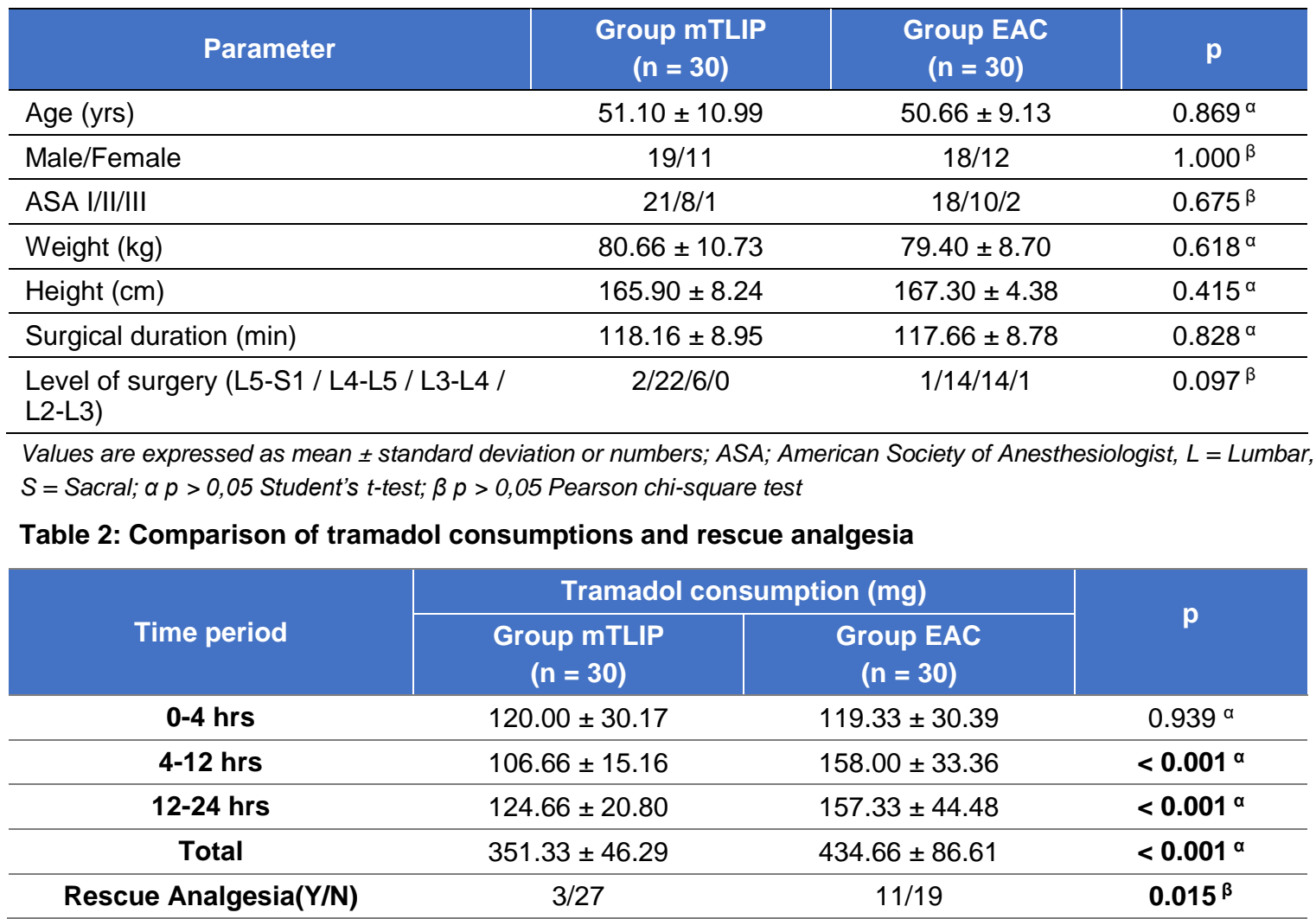

Values are expressed as mean \pm standard deviation; ${ }^{\alpha}$ Student's $t$-test; ${ }^{\beta}$ Fisher's exact Test

Table 3: Comparison of VAS scores

\begin{tabular}{c|c|c|c}
\multirow{2}{*}{ Time } & \multicolumn{2}{|c|}{ VAS } & $p^{\alpha}$ \\
\cline { 2 - 4 } & $\begin{array}{c}\text { Group mTLIP } \\
(\mathbf{n}=\mathbf{3 0})\end{array}$ & $\begin{array}{c}\text { Group EAC } \\
(\mathbf{n}=\mathbf{3 0})\end{array}$ & 0.245 \\
\hline 0 $\mathbf{h}$ & $2.76 \pm 2.11$ & $2.76 \pm 1.16$ & 0.369 \\
\hline 1st $\mathbf{~}$ & $2.76 \pm 1.27$ & $2.36 \pm 0.92$ & 0.366 \\
\hline 2nd $\mathbf{~}$ & $2.66 \pm 0.99$ & $2.73 \pm 0.69$ & $\mathbf{0 . 0 0 4}$ \\
\hline 8th $\mathbf{~}$ & $3.00 \pm 0.94$ & $3.43 \pm 0.67$ & $\mathbf{0 . 0 0 1}$ \\
\hline 12th $\mathbf{~}$ & $3.23 \pm 0.93$ & $5.43 \pm 0.50$ & $\mathbf{0 . 0 0 1}$ \\
\hline 24th $\mathbf{~}$ & $3.90 \pm 0.99$ & $4.76 \pm 0.72$ & $\mathbf{0 . 0 0 1}$
\end{tabular}

Values are expressed as mean \pm standard deviation; VAS = Visual analog scale score; $\alpha$ Student's $t$-test

Table 4: Comparison of side effects

\begin{tabular}{l|c|c|c}
\multicolumn{1}{c|}{ Side effect } & $\begin{array}{c}\text { Group mTLIP } \\
(\mathbf{n}=\mathbf{3 0})\end{array}$ & $\begin{array}{c}\text { Group EAC } \\
(\mathbf{n}=30)\end{array}$ & $p^{\alpha}$ \\
Urinary retention & 0 & 1 & 0.313 \\
\hline Constipation & 0 & 1 & 0.313 \\
\hline Nausea/Vomiting & 5 & 8 & 0.347 \\
\hline Pruritus & 2 & 3 & 0.640 \\
\hline
\end{tabular}

Values are expressed as numbers; $\alpha$ Fisher's exact test 
In contrast to these studies, a previous study comparing the TLIP block with wound site infiltration showed that the TLIP block had no superiority in patients who underwent a single-level discectomy. ${ }^{21}$ The TLIP block has also been used successfully in the management of low back pain. ${ }^{22}$

Hand et al. defined the TLIP block as an intervention method at the area between the multifidus and longissimus muscles to target the dorsal ramus of the thoracolumbar nerves. ${ }^{12}$ Spinal nerves exiting the intervertebral foramina are divided into two as ventral ramus and dorsal ramus. The ventral ramus spreads towards the abdominal wall, whereas the dorsal ramus spreads towards the posterior thoracolumbar region. The dorsal ramus crosses the costotransverse ligament to enter inside the erector spinae muscle, where it passes inside the muscle to end/terminate by providing sensorial innervation to the upper back area. As an approach that is different from the classical TLIP application, the modified TLIP block was developed in two separate studies by Ahiskalioglu et al. and Ueshima et al., who showed it to have a level of effectiveness to the classical block. ${ }^{13}, 23$ The advantages of the modified TLIP block include easier application of the injection in the medial-lateral direction between the longissimus and iliocostalis muscles and the fact that it eliminates the need for a neuraxial puncture. If the block needle' direction and visualization on USG cooperation can't be established, lateral-medial intervention may be caused a neuraxial puncture. In our study, the modified TLIP block was administered and its effectiveness in the postoperative period was demonstrated. In the present study, the pinprick test performed following administration of the block showed that the block was effective between T7 and L1.

A review of the literature shows that there are no studies comparing the modified TLIP block with any another analgesic technique. In our study, epidural analgesia at closure was less effective than the TLIP block in all periods except the early postoperative period (first postoperative $4 \mathrm{hrs}$ ). We associate the reason for this with the pre-emptive administration of the TLIP block and the fact that area blocks have a longer duration of effect than epidural blocks. In addition, we believe that a certain volume of the local anesthetic administered to the epidural area at closure passes to the surrounding tissues, thereby reducing its effectiveness. The modified TLIP block also makes it possible to avoid the possible side effects of central neuraxial interventions. We prefer to administer the modified TLIP block before surgery. The reason that performing the block before surgery was not to impair the sonographic anatomy and to obtain clearer images. In addition, since paravertebral muscle integrity is impaired after surgery, the distribution of local anesthetic drugs for the plane blocks may be affected.

The limitations of this study first included the fact that the modified TLIP block was applied before the surgery, which could have caused the pre-emptive analgesic effectiveness of the modified TLIP block to appear to a greater extent. The second limitation is the fact that the study was not designed as a double-blind study. Finally, the study sample size was determined based on the opioid requirements, which was the primary aim of the study. The TLIP block-related side effects may not have been fully identified with the small sample size. Further studies with a larger sample size may be needed.

\section{Conclusion}

In lumbar discectomy, the modified thoracolumbar interfascial plane block has been associated with less opioid consumption and better pain scores compared with the application of epidural anesthesia at closure in patients. We consider that the modified thoracolumbar interfascial plane block constitutes a suitable alternative for postoperative analgesia in lumbar discectomy, and should be used as a part of balanced analgesia.

\section{Authors' contribution}

ECC: Content of work, study design, data collection, manuscript writing, statistical analysis

ME: Study design, manuscript writing

AMY: Statistical analysis, manuscript writing

AA: Manuscript writing, critical revision

MEA: Statistical analysis, manuscript writing

NCK: Data collection, critical revision

\section{Conflicts of interest}

There are no conflicts of interest declared by any of the authors. 


\section{References}

1. Kim YK, Kang D, Lee I, Kim SY. Differences in the incidence of symptomatic cervical and lumbar disc herniation according to age, sex and national health insurance eligibility: a pilot study on the disease's association with work. Int J Environ Res Public Health. 2018 Sep 25;15(10):2094. [PubMed] DOI: 10.3390/ijerph15102094

2. Li Z, Yang H, Liu M, Lu M, Chu J, Hou S, et al. Clinical characteristics and risk factors of recurrent lumbar disk herniation: a retrospective analysis of three hundred twenty-one cases. Spine. 2018;43(21):1463-1469. [PubMed] DOI: 10.1097/BRS.0000000000002655

3. Jordon J, Konstantinou K, O'Dowd J. Herniated lumbar disc. BMJ Clin Evid. 2009(209):1118. [PubMed]

4. Deyo RA, Tsui-Wu YJ. Descriptive epidemiology of lowback pain and its related medical care in the United States. Spine. 1987;12(3):264-248. [PubMed] DOI: 10.1097/00007632-198704000-00013

5. Radhakrishnan K, Kitchy WJ, O'Fallon WM, Kurland LT. Epidemiology of cervical radiculopathy: A population-based study from Rochester, Minnesota, 1976 through 1990. Brain. 1994(117):325-335. [PubMed] DOI: 10.1093/brain/117.2.325

6. Wilson-Smith A, Chang N, Lu VM, Mobbs RJ, Fadhil M et al. Epidural steroids at closure after microdiscectomy/laminectomy for reduction of postoperative analgesia: systematic review and metaanalysis. World Neurosurg. 2018;110:e212-e221. [PubMed] DOI: 10.1016/i.wneu.2017.10.133

7. Crofford LJ. Chronic pain: where the body meets the brain. Trans Am Clin Climatol Assoc. 2015;126:167-183. [PubMed]

8. Bajwa SJ, Haldar R. Pain management following spinal surgeries: An appraisal of the available options. J Craniovertebr Junction Spine. 2015;6(3):105-110. [PubMed] DOI: 10.4103/0974-8237.161589

9. Dunn LK, Durieux ME, Nemergut EC. Non-opioid analgesics: Novel approaches to perioperative analgesia for major spine surgery. Best Pract Res Clin Anaesthesiol. 2016;30(1):79-89. [PubMed] DOl: 10.1016/i.bpa.2015.11.002

10. Ahiskalioglu A, Yayik AM, Doymus O, Selvitopi K, Ahiskalioglu EO, Calikoglu C, et al. Efficacy of ultrasoundguided modified thoracolumbar interfascial plane block for postoperative analgesia after spinal surgery: a randomizedcontrolled trial. Can J Anaesth. 2018;65(5):603-604. [PubMed] DOI: 10.1007/s12630-018-1051-0

11. Yayik AM, Cesur S, Ozturk F, Ahiskalioglu A, Ay AN, Celik $E C$, et al. Postoperative analgesic efficacy of the ultrasound-guided erector spinae plane block in patients undergoing lumbar spinal decompression surgery: a randomized controlled study. World Neurosurg. 2019;126:e779-e785. [PubMed] DOI: 10.1016/i.wneu.2019.02.149

12. Hand WR, Taylor JM, Harvey NR, Epperson TI, Gunselman $\mathrm{RJ}$, Bolin ED, et al. Thoracolumbar interfascial plane (TLIP) block: a pilot study in volunteers. Can J Anaesth. 2015;62(11):1196-1200. [PubMed] DOI: 10.1007/s12630015-0431-y

13. Ahiskalioglu A, Alici HA, Selvitopi K, Yayik AM. Ultrasonography-guided modified thoracolumbar interfascial plane block: a new approach. Can J Anaesth. 2017;64(7):775-776. [PubMed] DOI: 10.1007/s12630-017$\underline{0851-y}$

14. Lotfinia I, Khallaghi E, Meshkini A, Shakeri M, Shima M, Safaeian A. Interaoperative use of epidural methylprednisolone or bupivacaine for postsurgical lumbar discectomy pain relief: a randomized, placebo-controlled trial. Ann Saudi Med. 2007;27(4):279-283. [PubMed] DOI: 10.5144/0256-4947.2007.279

15. Seal DD, Loken RG, Hurlbert RJ. A surgically placed epidural catheter in a patient with spinal trauma. Can J Anaesth. 1998 Feb;45(2):170-174. [PubMed] DOI: 10.1007/BF03013258

16. Cesur S, Yayik AM, Ozturk F, Ahiskalioglu A. Ultrasoundguided low thoracic erector spinae plane block for effective postoperative analgesia after lumbar surgery: report of five cases. Cureus. 2018;10(11):e3603. [PubMed] DOI: 10.7759/cureus. 3603

17. Melvin JP, Schrot RJ, Chu GM, Chin KJ. Low thoracic erector spinae plane block for perioperative analgesia in lumbosacral spine surgery: a case series. Can J Anaesth. 2018;65(9):1057-1065. [PubMed] DOI: 10.1007/s12630$\underline{018-1145-8}$

18. Chin KJ, Lewis S. Opioid-free analgesia for posterior spinal fusion surgery using erector spinae plane (esp) blocks in a multimodal anesthetic regimen. Spine. 2019 Mar 15;44(6):E379-E383. [PubMed] DOI: 10.1097/BRS.0000000000002855

19. Ammar MA, Taeimah M. Evaluation of thoracolumbar interfascial plane block for postoperative analgesia after herniated lumbar disc surgery: A randomized clinical trial. Saudi J Anaesth. 2018;12(4):559-564. [PubMed] DOI: 10.4103/sja.SJA_177_18

20. Ueshima H, Ozawa T, Toyone T, Otake H. Efficacy of the thoracolumbar interfascial plane block for lumbar laminoplasty: a retrospective study. Asian Spine J. 2017;11(5):722-725. [PubMed] DOI: 10.4184/asj.2017.11.5.722

21. Ince I, Atalay C, Ozmen O, Ozturk V, Hassan M, Aksoy M, et al. Comparison of ultrasound-guided thoracolumbar interfascial plane block versus wound infiltration for postoperative analgesia after single-level discectomy. J Clin Anesth. 2019 Sep;56:113-114. [PubMed] DOI: 10.1016/i.jclinane.2019.01.017

22. Ahiskalioglu A, Yayik AM, Celik EC, Aydin ME, Uzun G. Ultrasound guided modified thoracolumbar interfascial plane block for low back pain management. J Clin Anesth. 2019;54:138-139. [PubMed] DOI: 10.1016/j.jclinane.2018.11.010

23. Ueshima $H$, Otake $H$. Ultrasound-guided "lateral" thoracolumbar interfascial plane (TLIP) block: A cadaveric study of the spread of injectate. J Clin Anesth. 2017;40:54. [PubMed] DOI: 10.1016/i.jclinane.2017.04.013 